\title{
Review of Extracorporeal Membrane Oxygenation and Dialysis-Based Liver Support Devices for the Use of Nephrologists
}

\author{
Tamim Hamdi Biff F. Palmer \\ Department of Internal Medicine, Division of Nephrology, UT Southwestern, Dallas, TX, USA
}

\begin{abstract}
Keywords
Extracorporeal membrane oxygenation - Continuous renal replacement therapy $\cdot$ Molecular adsorbent recirculation support · Prometheus liver support device · Single pass albumin dialysis - Selective cytopheretic device
\end{abstract}

\begin{abstract}
Acute kidney injury in the intensive care unit (ICU) is a manifestation of an underlying severe illness that commonly involves other organ systems. Pulmonary, cardiac, and hepatic failures are the most prevalent. This article provides a simplified review of the technical aspects of extracorporeal cardiopulmonary and liver support devices used in the adult ICU patient, as well as a summary of the most relevant and upto-date clinical evidence that supports their use.
\end{abstract}

(c) 2017 S. Karger AG, Basel

\section{Introduction}

The nephrologist commonly encounters patients with multiple organ failure in the intensive care unit (ICU), as acute kidney injury (AKI) affects at least one third of this patient population [1-3]. In an ICU patient with AKI, the number of failed organ systems is reported to be as high as 2.9 [4], with respiratory failure being the most prevalent $(67 \%)$, followed by cardiovascular (48\%) and liver failure (19\%) [4]. As novel support devices for failed or- gan systems are continuously being developed, the nephrologist faces the challenge of understanding the technical and clinical aspects of their use. The aim of this paper is to provide a simplified overview of those devices used in adult patients. This article will not discuss the use of mechanical ventilation, plasma exchange, and intracorporeal cardiac assist devices use outside of the ICU but will briefly discuss a dialysis circuit-based experimental device for sepsis.

\section{Extra-Corporeal Membrane Oxygenation}

Extra-corporeal membrane oxygenation (ECMO) is primarily used to provide cardiopulmonary support. Nearly 2 decades after Gibbon [5] pioneered the heart-lung machine in 1953, an ECMO-like circuit was successfully used by Hill et al. [6]. Bartlett et al. [7] eventually introduced the modern ECMO design in 1975 to save the life of the famous "Baby Esperanza" who became the first neonatal ECMO survivor. The number of ECMO cases has nearly tripled over the last 2 decades [8] with an exponential increase since 2009 when the conventional ventilatory support vs. extracorporeal membrane oxygenation for severe adult respiratory failure (CESAR) randomized trial [9] and the Australia and New Zealand Extracorporeal Membrane Oxygenation (ANZ ECMO) observational study [10] were published. The nephrologist is likely to encounter such therapies when providing renal care in the ICU.

\section{KARGER}

(c) 2017 S. Karger AG, Basel

E-Mail karger@karger.com

www.karger.com/ajn
Tamim Hamdi, MD

Department of Internal Medicine, Division of Nephrology

University of Texas Southwestern, 5323 Harry Hines Blvd

POB 2 HQ1.202E, Dallas, TX 75390 (USA)

E-Mail tamim.hamdi@utsouthwestern.edu 
Fig. 1. VV and veno-veno-arterial (VVA) ECMO circuits: VV ECMO: deoxygenated blood (blue) is drained through the right femoral vein and retuned through the right internal jugular vein. Insert: dual-lumen single cannula drains the superior and inferior venae cava (blue arrows) and returns the oxygenated blood (red arrow) through a separate lumen. If cardiac support is required, an additional cannula can be inserted into the femoral artery (broken line) to a form a VVA ECMO circuit.

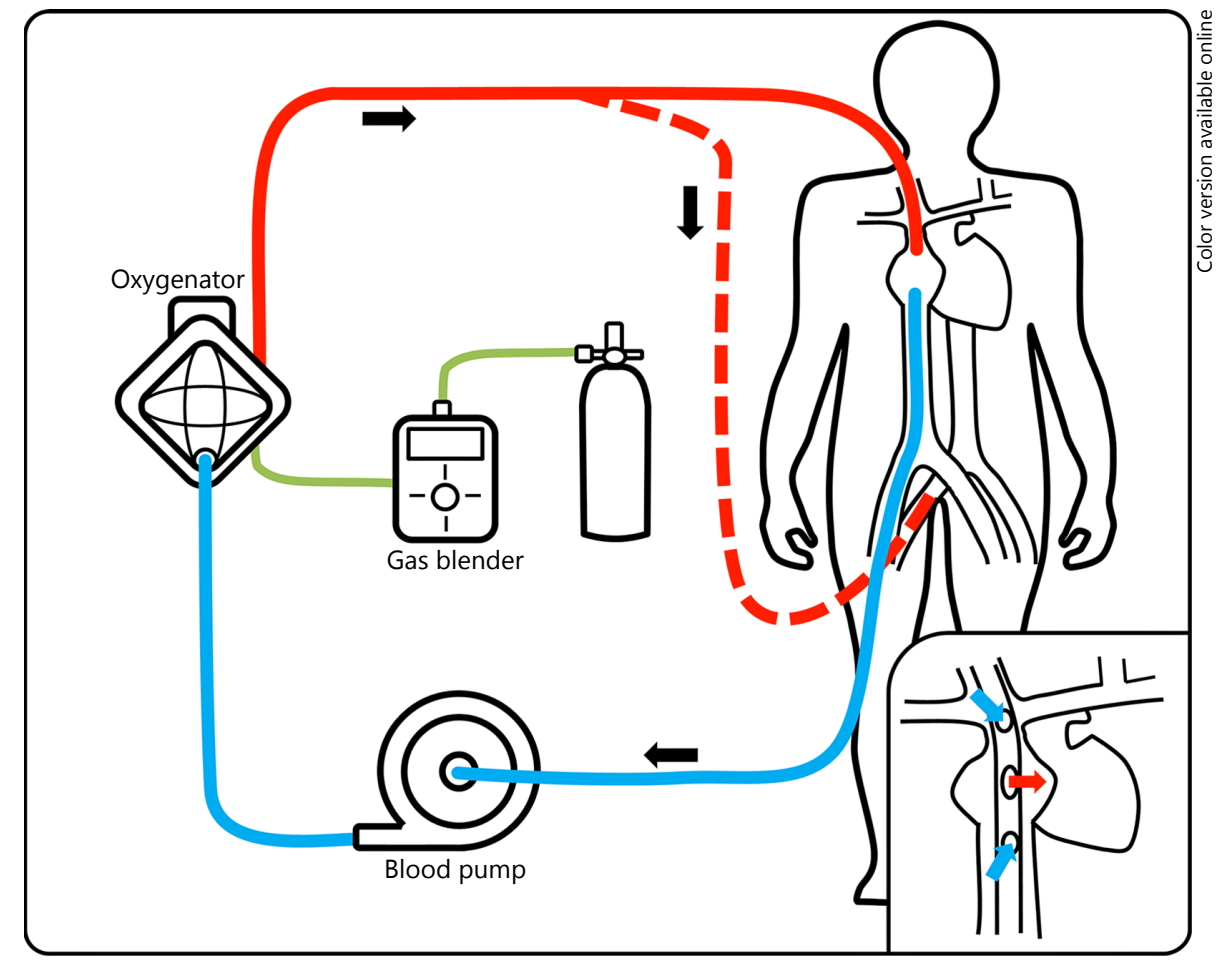

\section{Venous-Venous ECMO}

Venous-venous ECMO (VV ECMO) provides pure respiratory support through blood oxygenation and carbon dioxide $\left(\mathrm{CO}_{2}\right)$ removal. It does not provide circulatory support. A related technique is available for isolated $\mathrm{CO}_{2}$ removal (extracorporeal $\mathrm{CO}_{2}$ removal-ECCO${ }_{2} \mathrm{R}$ ) and is used as an adjunct to advanced ventilation strategies. VV ECMO use is indicated in isolated refractory hypoxemia in the setting of severe acute respiratory distress syndrome (ARDS) despite conventional mechanical ventilation [11]. Less common indications with scarce evidence include acute pulmonary embolism, primary graft failure following lung transplant, and steroid-resistant acute eosinophilic pneumonia. Eligible patients should generally have a reversible etiology of respiratory failure and absence of other end-stage organ dysfunction. There are no absolute contraindications to the initiation of $\mathrm{VV}$ ECMO and selection criteria largely depend on individual cases and local expertise. In a large cohort of ECMO patients with respiratory failure $(78 \%$ receiving pure $\mathrm{VV}$ mode), the longer duration of pre-ECMO mechanical ventilation was associated with a higher mortality rate, although this relationship disappeared in the most recent subgroup of patients (2002-2006) who received a median of just $42 \mathrm{~h}$ of pre-ECMO mechanical ventilation [12]. This suggests a possible shift in practice with the earlier initiation of ECMO, although some relevant ventilation parameters were not reported. Other studies suggest a cutoff of 7 days of mechanical ventilation with elevated airway pressures and oxygen requirements, which might denote the onset of the proliferative phase of ARDS with potential lung fibrosis $[11,13]$. Thus, the duration of preECMO mechanical ventilation remains a consideration rather than a contraindication to ECMO initiation [12]. Other relative contraindications associated with a poor prognosis include older age, cardiac arrest, high risk or current bleeding, immunocompromised state, morbid obesity, and central nervous system dysfunction, among others [14]. The Respiratory Extracorporeal Membrane Oxygenation Survival Prediction (RESP) score [15] was derived from the largest cohort of ARDS patients to date and can be used to predict the mortality rate of patients already receiving VV ECMO (calculator available at www.respscore.com).

The typical VV ECMO circuit is shown in Figure 1. The drainage cannula is usually located in the inferior vena cava through the femoral vein and the return cannula in the superior vena cava through the right internal jugular vein. A dual-lumen single internal jugular cannula (Avalon Elite, Avalon Laboratories, Rancho Dominguez, CA, USA) is available and provides the advantages of occupying one anatomical site, reducing blood recir- 
culation, and allowing patient ambulation $[16,17]$ but at the expense of more complicated placement requiring direct visualization and operator expertise. Patients occasionally develop cardiac decompensation and require the insertion of an additional return cannula into the arterial system. This configuration is termed veno-veno-arterial ECMO [18].

After setting up the circuit and providing adequate systemic anticoagulation (usually with heparin), the ECMO blood flow is quickly increased to match the patient's cardiac output and target an arterial oxygen saturation target of more $80 \%$. The sweep gas flow rate that determines $\mathrm{CO}_{2}$ removal is adjusted to reach a normal $\mathrm{pH}$ value and $\mathrm{PCO}_{2}$ level. Ventilator settings are reduced to allow lung rest and healing. Details regarding the management of VV ECMO are beyond the scope of this article, but one common scenario is relevant to the nephrologist. A low pressure in the drainage cannula $(<-300 \mathrm{~mm} \mathrm{Hg})$ can manifest as line pulsations ("chattering") and results from circuit-related causes (small cannula size, cannula malposition or kinking, mismatched blood flow, etc.) or hypovolemia. In the latter case, the rate of ultrafiltration or diuresis should be reduced. Bleeding and thrombosis are among the most common complications [19] and their respective risks should be balanced against each other based on individual cases. Weaning from VV ECMO is achieved by gradually reducing the sweep gas flow rate.

Evidence to support the use of VV ECMO in adults is limited. A randomized trial in 1979 failed to show mortality benefits when VV ECMO was added to mechanical ventilation [20]. The mortality rates were high in both groups (90\%) likely secondary to the primitive technology and different practices in the ICU at that time. A second trial in 1993 failed to show survival benefits with $\mathrm{ECCO}_{2} \mathrm{R}$ [21]. The only randomized trial in the modern era is the CESAR trial [9], where 90 adult patients with ARDS received conventional ventilation in their local study center and another 90 were transferred to a tertiary care center with ECMO capabilities. The primary endpoint of death or severe disability at 6 months was significantly less in the group transferred to the ECMO center (63 vs. $47 \%, p=$ 0.03 ). Although this trial suggests a potential survival benefit, the results may have been confounded by the absence of standardized ventilation protocols in the control group (lung protective ventilation strategies were recommended but not imposed). In addition, only $75 \%$ of patients transferred to the ECMO center received ECMO with the rest improving with conventional ventilation, thereby suggesting possible benefits resulting from the expertise in the specialized ECMO medical center.

Extracorporeal Membrane Oxygenation and Dialysis-Based Liver Support Devices
Shortly after the CESAR trial was published, the ANZ ECMO observational trial reported a survival rate of $71 \%$ to hospital discharge in 68 patients treated with ECMO for influenza A (H1N1)-related ARDS [9]. This outcome favorably compares to the $58 \%$ survival to discharge or transfer in adults with VV ECMO based on the 2016 extra-corporeal life support organization registry [8]. Thus, this modality remains an invaluable and potentially lifesaving option in patients who otherwise have an estimated mortality of $>80 \%$.

The Extracorporeal Membrane Oxygenation for Severe Acute Respiratory Distress Syndrome trial [22] is currently recruiting patients and will be examining the effect of using VV ECMO after 3-6 h of poorly evolving ARDS.

\section{Veno-Arterial ECMO}

Veno-arterial ECMO (VA ECMO) is similar to the heart-lung machine and provides cardiac and pulmonary support for patients in cardiogenic shock. Survivors of VA ECMO recover or transition to cardiac transplantation or implant of a long-term left ventricular assist device (LVAD). Central VA ECMO uses transthoracic cannulas typically inserted during cardiac bypass in patients who need immediate postoperative support. Deoxygenated blood is drained from the right atrium and returned to the aorta. Peripheral VA ECMO uses a drainage cannula in a major vein (usually femoral) to drain the deoxygenated blood and a return cannula in a major artery (femoral artery in adults, carotid artery in children). The oxygenated blood that is returned through the femoral arterial cannula will flow in a retrograde direction through the descending aorta and mixes at some point with the poorly oxygenated blood pumped by the left ventricle (LV). In the presence of residual LV function, this configuration might lead to upper body (differential) hypoxemia. To overcome this problem, the ECMO blood flow can be increased to propel the blood upwards and/or the femoral return cannula switched to the carotid or axillary arteries. Alternatively, an extra cannula carrying oxygenated blood can be inserted into the right atrium or even connected to a preexisting dialysis catheter [23]. This configuration is termed veno-arterio-venous ECMO and provides improved coronary and cerebral perfusions, potentially reduces ventilator settings, and reduces right ventricular (RV) afterload through the attenuation of pulmonary artery hypoxic vasoconstriction. In order to prevent distal lower limb ischemia, an additional cannula is usually inserted into the femoral artery to provide anterograde flow (Fig. 2) or the posterior tibial artery to provide retrograde flow [24, 25]. 
Fig. 2. VA and veno-arterio-venous (VAV) ECMO circuits: VA ECMO: peripheral cannulation where deoxygenated blood (blue) is drained through the right internal jugular vein and retuned through the left femoral artery. Blood flow through the descending aorta is retrograde (upward red arrow). Distal limb ischemia is prevented using an additional cannula for anterograde limb perfusion (downward red arrow). If upper body hypoxia develops, a return cannula can be inserted into the right atrium through the right internal jugular vein (broken line) to form a VAV ECMO circuit.

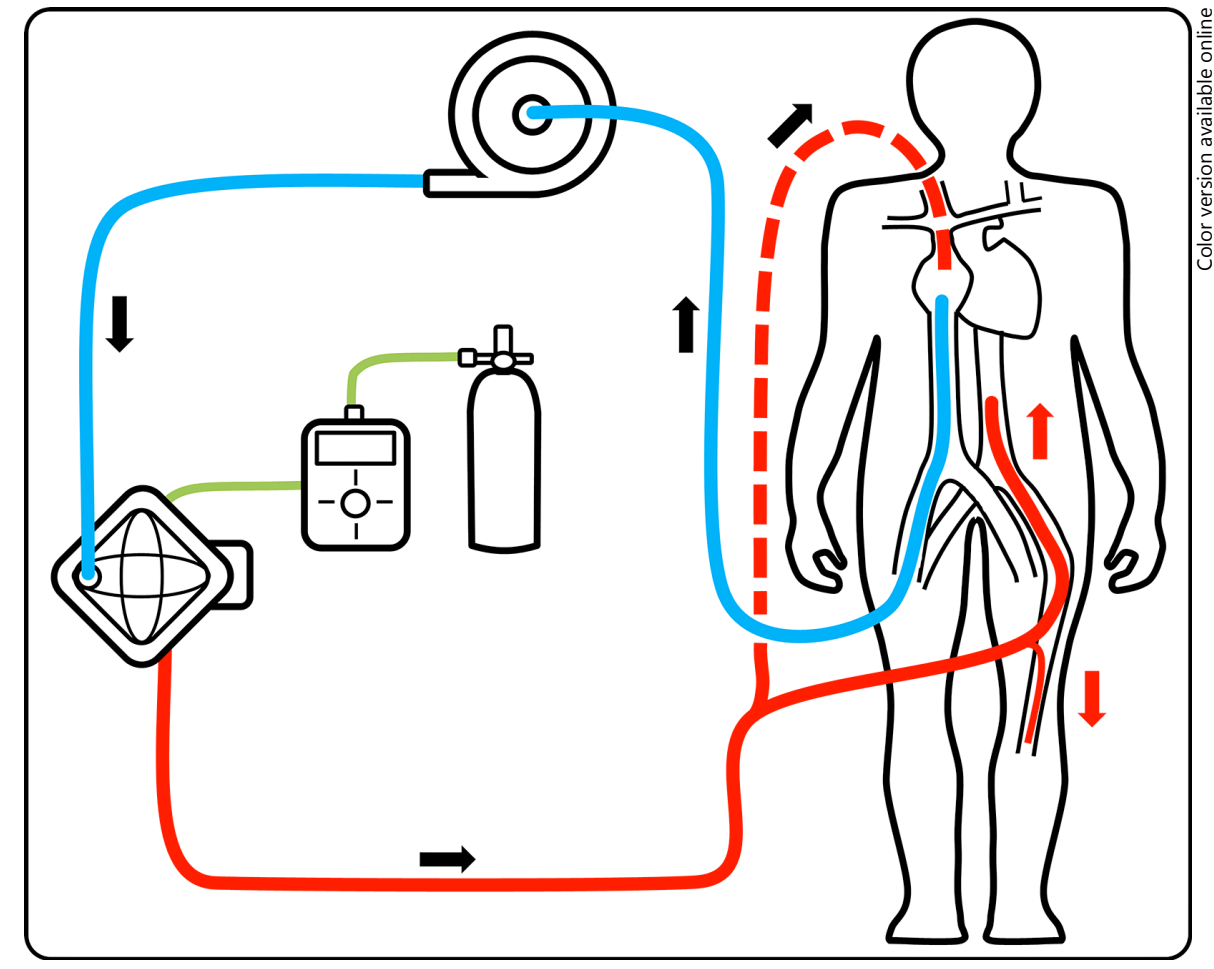

After setting up the circuit and providing adequate systemic anticoagulation, the ECMO blood flow is titrated to target adequate tissue perfusion and drain the LV. The LV residual function and recovery can be assessed daily using a bedside "mini" transesophageal echocardiogram and by observing for arterial wave pulsation. A persistently poor LV function will result in LV distention, poor recovery, and inability to open the aortic valve with increased risk for LV thrombus formation. This issue can be managed with inotropes, after-load reduction, surgical LV decompression [26, 27], or using an intra-aortic balloon pump [28] or Impella microaxial flow device in conjunction with ECMO. Weaning from VA ECMO is achieved by gradually reducing the ECMO blood flow. Based on the 2016 extra-corporeal life support organization registry [8], survival to discharge or transfer in adults treated with VA ECMO is $41 \%$, which is lower than that of patients receiving VV ECMO.

\section{ECMO and the Kidney}

AKI is a common complication in the adult ECMO patients. Using the risk, injury, failure, loss, and end stage or AKI Network criteria, 2 single-center studies showed an AKI incidence of more than $80 \%$ with close to half of affected patients requiring renal replacement therapy (RRT) $[29,30]$. In a third study of 200 adult patients, 120 patients required RRT within the first week of ECMO [31]. In all 3 studies, the mortality rate was almost threefold higher for patients who received RRT compared to those who did not and the severity of kidney injury was an independent predictor of death. A recent meta-analysis of 21,624 adult and pediatric ECMO patients showed that mortality rate was $89 \%$ higher for patients who received concomitant RRT compared to those who did not [32]. In this study, the mortality rates in both groups (with vs. without RRT) rose in parallel to the severity of the underlying illness, with the highest mortality observed in the extracorporeal cardiopulmonary resuscitation group followed by ECMO for cardiac and then pulmonary indications. These observations led the authors to conclude that the higher mortality rates observed in the RRT group were attributed to the severity of the underlying illness rather than the use of RRT itself [32].

Both patient and ECMO-related factors contribute to the development of AKI. Patients initiated on ECMO are severely ill and often have a preexisting impairment in hemodynamics and/or systemic inflammation [33]. Many patients are already receiving RRT prior to ECMO initiation [31]. The pathogenesis of AKI in this setting is related to a number of ECMO-related factors to include impaired renal perfusion due to non-pulsatile flow (exclusive to patients with reduced cardiac function on VA ECMO), dis- 
ruption of the renin-angiotensin-aldosterone axis, a general pro-inflammatory state induced by blood shear stress and exposure to the non-biologic oxygenator membrane, effects of cardio-renal and pulmonary-renal cross talk, circuit-related complications such as pigment-induced nephropathy caused by hemolysis or limb ischemia, and finally rare complications such as thrombotic or air emboli as well as aortic dissection involving the renal arteries. This topic has been the subject of a recent review [34].

Fluid overload (FO) in the general ICU patient with AKI is independently associated with higher mortality rate [35]. FO similarly compromises the cardiac and/or pulmonary functions in the ECMO patient and therefore current guidelines recommend achieving and maintaining euvolemia once the hemodynamics are stabilized [11]. An international survey reported that treatment and prevention of $\mathrm{FO}$ are critically important indications for using RRT in conjunction with ECMO (43 and 16\%, respectively) [36]. While the majority of the available studies included pediatric patients, Schmidt et al. [37] reported data on 172 adult ECMO patients of whom 103 required RRT and found that FO at day 3 of ECMO use was a strong and independent predictor of 90 -day mortality even after adjusting for severity of illness and other confounding factors. This relation occurred in patients with and without RRT and the median daily fluid balance among patients who received RRT was 2.9 times higher for non-survivors compared to survivors (+558 vs. +191 $\mathrm{mL} /$ day). Until randomized controlled trial specifically examines various fluid balance strategies [38], the current data suggest that achieving euvolemia in ECMO patients is a reasonable goal and might confer clinical benefits.

A subset of patients on continuous RRT (CRRT) receiving respiratory support using ECMO might develop respiratory acidosis during weaning especially with the standard use of lung-protective ventilation and resulting permissive hypercapnia in patients with ARDS. The pros and cons of hypercapneic respiratory acidosis and the value of its correction using bicarbonate are controversial $[39,40]$, but the ICU team might desire a target $\mathrm{pH}$ of 7.3 or higher as per the landmark low tidal volume trial [41]. Note that the study protocol allowed the administration of sodium bicarbonate if the $\mathrm{pH}$ remained below target despite ventilator manipulations [41], and respiratory acidosis has been implicated as a cause of patient-ventilator asynchrony ("double triggering"), which might negate the expected benefits of such strategy [40,42]. Close communication between the nephrologist and the ICU team is imperative to provide a smooth transition during this critical phase in the care of the ECMO patient and the

Extracorporeal Membrane Oxygenation and Dialysis-Based Liver Support Devices nephrologist might be able to modify the CRRT prescription on a case-by-case basis.

Renal outcomes in surviving adult patients who received ECMO and CRRT are unclear. Pediatric data show that the overwhelming majority of survivors recover kidney function and those who do not usually have another underlying etiology such as glomerulonephritis or primary renal disease $[43,44]$.

RRT can be provided using a "home-made" circuit or more commonly using a conventional CRRT machine (Fig. 3a). In the first option, a hemofilter is placed on the positive pressure side of the ECMO circuit to facilitate blood flow, a standard intravenous fluid pump provides negative pressure on the effluent side of the hemofilter, and a urometer (or scale) measures the effluent rate (or weight). Dialysate and/or replacement fluid can be added to provide solute clearance $[45,46]$. The major concerns limiting the use of this approach are ultrafiltrate volume inaccuracy [47], absence of filter pressure monitoring, and poor solute clearance in case of isolated ultrafiltration or when this setup is used outside the pediatric realm where the higher flows required to attain meaningful clearance will be limited by the inherent pump capability.

Standard CRRT is provided using a separate vascular access or by connecting the CRRT machine to the ECMO circuit. Patients already receiving CRRT before ECMO initiation should continue to use their current catheter. Patients initiated on CRRT while on ECMO have the option of either connecting the CRRT device to the ECMO circuit or having a new dialysis catheter placed. The latter option carries a risk of bleeding due to the systemic anticoagulation and occupying an access site that might be valuable for future needs. The decision is largely dictated by the physician and nursing's expertise and level of comfort. The ideal setup of a CRRT machine connected to the ECMO circuit has the CRRT inlet line connected post ECMO pump where the pressure is positive to prevent air entrainment, although the positive pressure will trigger the access disconnection alarm on some CRRT machines (Fig. 3b). Many CRRT machines can circumvent this issue by allowing the customization of alarm parameters. For example, the commonly used Prismaflex (Gambro, Sweden) CRRT machine allows the user to select a positive pressure access when the treatment is initiated without the need for any additional user input (software version 7.11). The CRRT outlet line should be connected before the oxygenator to trap air emboli and to avoid delivering deoxygenated blood to the patient $[33,46]$. This setup is limited by the ECMO console in use. For example, the Maquet Rotaflow permanent life support system (Maquet Cardiopulmonary AG, 


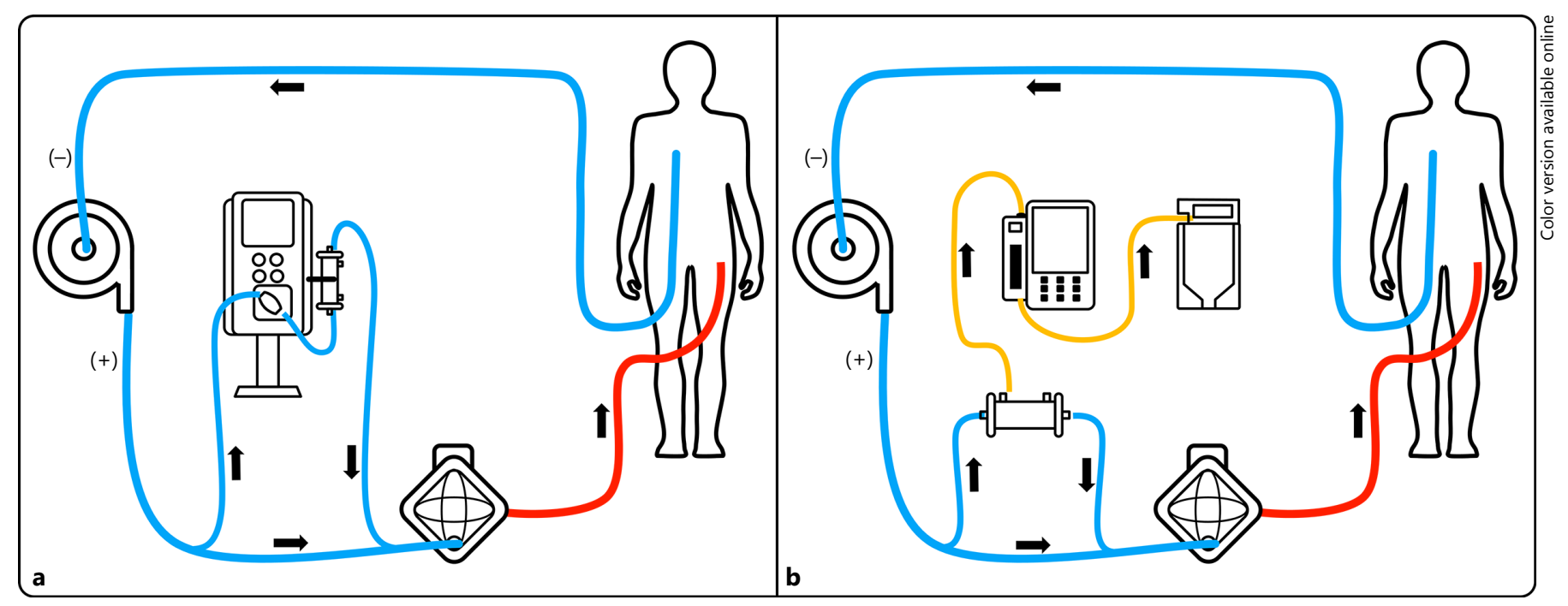

Fig. 3. CRRT setups: a Ideal CRRT setup using a conventional CRRT machine with intake and return lines between the ECMO pump and the oxygenator to prevent air entrainment and bypass of the oxygenator. The applicability of this configuration depends on the type of

Hirrlingen, Germany) uses a separate pump and oxygenator, thus allowing the connection of CRRT using the described configuration. By contrast, in the newer and compact Maquet Cardiohelp heart lung support system, the blood pump and oxygenator are physically integrated leaving no room for use of CRRT in that location. In this case, the CRRT device can be connected to the drainage cannula either while the patient is receiving ECMO or preferably while setting up the ECMO tubing if CRRT is anticipated. Systemic anticoagulation during ECMO is adequate to reduce the risk of CRRT circuit clotting. In the rare ECMO patient without anticoagulation, clotting in the CRRT circuit is managed similarly to the general ICU patient by increasing the blood flow and/or the pre-filter replacement fluid rate, or utilizing regional citrate anticoagulation protocol if a reliable protocol is available.

\section{Other Extracorporeal Circulatory Support Devices}

The CentriMag (Thoratec, Pleasanton, CA, USA) is an extracorporeal magnetically levitated centrifugal pumpbased, short-term assist device that is FDA-approved for use in acute RV failure up to 30 days. CentriMag is commonly used as a bridge-to-decision and for RV failure that develops during or after placement of a LVAD. Such patients usually have a high mortality rate, and temporary use of this device improves right and left-sided hemody-
ECMO machine used. b Home-made CRRT setup using a hemofilter, fluid pumps, and an urometer. Replacement fluid and/or dialysate can be added to the circuit. + and - denote positive and negative pressures respectively. namics facilitating fluid removal via CRRT [48-50]. Offlabel use as an LVAD and for extended durations has been reported [51, 52].

The TandemHeart (CardiacAssist, Pittsburgh, PA, USA) is a percutaneous ventricular assist device that drains the left atrium through a trans-septal cannula inserted via the femoral vein and pumps the blood back via the femoral artery. This device can provide a flow up to $5 \mathrm{~L} / \mathrm{mn}$ and is FDA-approved for left ventricular support for up to $6 \mathrm{~h}$. Mortality rate is similar, while limb ischemia and bleeding are more common when compared to the intra-aortic balloon pump in patients with cardiogenic shock after an acute myocardial infarction [53]. The TandemHeart has also been used for RV support [54, 55].

\section{Extra-Corporeal Liver Support}

In the United States, acute liver failure affects 2,000 persons annually [56] with close to half of the cases attributed to acetaminophen toxicity [57]. AKI develops in $75 \%$ of cases caused by acetaminophen overdose and in $30 \%$ of all other cases $[58,59]$. As only $45 \%$ of patients survive without a liver transplantation [57], various strategies were developed to provide temporary liver support as a bridge to recovery or transplantation. Bioartificial devices support synthetic and excretory liver functions [60, 61 ], while acellular ones only support the latter and are 


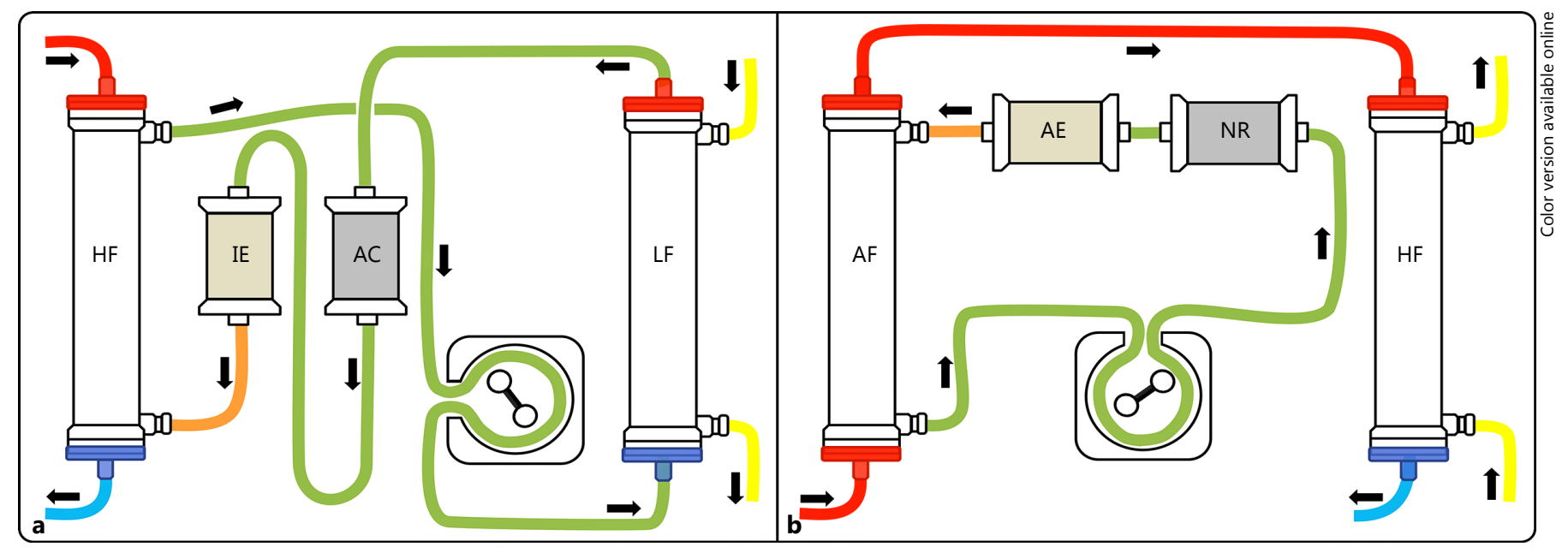

Fig. 4. Mars and Prometheus liver support devices: a MARS liver support device. HF, high flux dialyzer; LF, low flux dialyzer; AC, activated charcoal; IE, ion exchange. b Prometheus liver support device. AF, albumin filter; NR, neutral resin; AE, anion exchange.

discussed below. The 3 most commonly used techniques include molecular adsorbent recirculation support (MARS), fractionated plasma separation and adsorption (FPSA), and single-pass albumin dialysis (SPAD). These techniques share the concept of removing protein-bound toxins using adsorption to albumin in order to reduce the severity of organ dysfunction (renal, immune, and cerebral), improve local and systemic hemodynamics by clearing vasoactive substances, and provide a healthier milieu for hepatocyte regeneration $[60,62]$.

\section{Molecular Adsorbent Recirculation Support}

The concept of albumin recirculation was introduced in 1993 and used clinically in 1996 [63]. The MARS console (Gambro, Sweden) connects to the Prismaflex CRRT machine to provide both hepatic and renal support. The patient's blood enters the albumin-impregnated high flux polysulfone filter and is dialyzed against $20 \%$ albumin solution. The molecular cutoff of this filter is $50 \mathrm{kDa}$ and therefore does not allow the filtration of patient's albumin across the membrane. The albumin solution saturated with toxins passes across a low-flux filter for clearance of water-soluble molecules using a standard dialysate and then is reactivated using an activated charcoal column and anion exchangecartridge(Fig.4a).MARSisFDA-approved for use in protein-bound drug poisoning and treatment of hepatic encephalopathy in decompensated chronic liver disease. Off-label use in many conditions has been reported with variable success rates [60]. After years of conflicting data, the RELIEF study group randomized 189 pa-
The green segments in both circuits indicate the presence of albumin-bound wastes. The orange segments indicate the removal of albumin-bound wastes. The yellow segments indicate the dialysate. The blue segments indicate the blood return line to the patient.

tients with acute-on-chronic liver failure to standard medical therapy with or without MARS. The primary endpoint of liver transplantation-free survival within 28 days was similar between groups, and a nonsignificant improvement in hepatic encephalopathy and bilirubin levels was observed in the MARS group [64].

\section{Prometheus Liver Support Device}

The concept of FPSA was introduced in 1999 [65] and is utilized by the Prometheus machine (Fresenius Medical Care, Bad Homburg, Germany). Unlike MARS, which relies on an exogenous albumin solution, the Prometheus machines allows the patient's own albumin to enter the FPSA circuit facilitated by the AlbuFlow filter's molecular cutoff of $250 \mathrm{kDa}$. Albumin is reactivated and returned to the circulation (Fig. 4b). The HELIOS study group randomized 145 patients with acute-on-chronic liver failure to standard medical therapy with or without Prometheus. The mortality rate at 28 and 90 days was similar, and a reduction in serum bilirubin was observed only in the intervention group. A subgroup analysis showed improved survival among patients with a MELD score greater than 30 [66]. The Prometheus machine is not available in the United States. In a small study, Prometheus was compared to MARS and reported to achieve higher clearance of protein-bound solutes [67]. Given the current evidence, these devices are not routinely used but rather considered in selected individuals with severe liver failure (MELD $>30)$ as bridge to transplantation. 
Fig. 5. Selective cytopheretic device (SCD) where dialyzed blood circulates through the dialysate ports. Cit, citrate; $\mathrm{Ca}$, calcium.

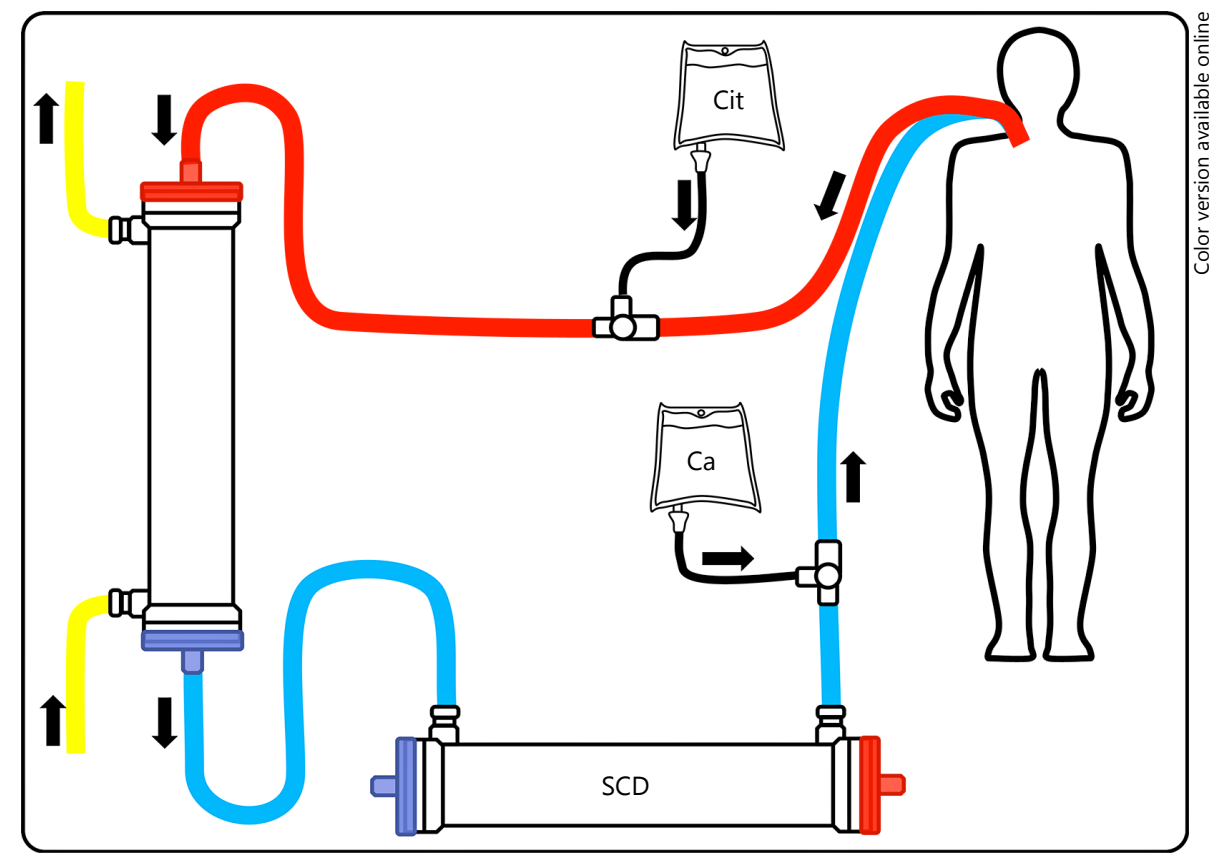

\section{Single-Pass Albumin Dialysis}

SPAD involves a typical CRRT setup but with albumin added to the dialysate. An in-vitro study reported that SPAD using $4 \%$ dialysate albumin removed significantly more bilirubin and similar bile acids when compared to MARS running in CVVHDF mode. The financial cost of a 7-h session was lower with SPAD despite excluding the cost of the MARS monitor [68]. In a recent trial [69], 32 patients with liver failure completed $69 \mathrm{cy}-$ cles of MARS (blood flow $=$ albumin flow $=150 \mathrm{~mL} / \mathrm{mn}$, dialysate flow $=2 \mathrm{~L} / \mathrm{h}$ ) and SPAD (blood flow $=50 \mathrm{~mL} /$ $\mathrm{mn}, 4 \%$ albumin dialysate flow $=0.7 \mathrm{~L} / \mathrm{h}$ ), each lasting 7-8 h. Both modalities reduced bilirubin levels to the same extent, but only MARS increased the albuminbinding capacity. The latter can be explained by the lower clearance of bile acids with SPAD probably due to lower dialysis dose $[68,70]$ and the occupation of albuminbinding sites by the heat stabilizer octanoate. Octanoate is removed using the MARS system as albumin passes through the activated charcoal and ion exchange cartridges. Hepatic encephalopathy, Glasgow Coma Scale, and Ramsay scores did not improve in either group. With regards to the dose of albumin, one case report showed that dialysate albumin as low as $1.85 \%$ resulted in similar removal of bilirubin when compared to 5\% [71]. Finally, SPAD was reported to increase the elimination of albumin-bound drugs especially with the presence of AKI that would reduce their renal drug elimination [72-74]. The in-vitro study by Churchwell et al. [74] was the most controlled as it compared the effect of various blood flows (180-270 mL/mn), dialysate flows ( $1-4 \mathrm{~L} / \mathrm{h})$, dialysate albumin concentrations $(0,2.5$, and $5 \%)$, and dialyzers $\left(1.5 \mathrm{~m}^{2}\right.$ polysulfone and $0.6 \mathrm{~m}^{2}$ acrylonitrile 69$)$ on the clearance of phenytoin, valproic acid, and carbamazepine. The study did not include any hemadsorption techniques that would confound the results and the authors measured the drug concentrations in the blood and the spent dialysate. The highest clearances (for carbamazepine and valproic acid) were achieved using the combination of $5 \%$ albumin dialysate with the large polysulfone dialyzer, while the blood and dialysate flows did not consistently and significantly affect the clearances.

\section{CRRT-Based Therapy for Septic Shock}

The selective cytopheretic device (SCD) is a cartridge resembling a polysulfone hemofilter that is thought to preferentially bind then deactivate neutrophils and monocytes in the presence of a low calcium milieu [75, 76]. The SCD is mounted on a CRRT machine circuit in series with the conventional hemodialyzer. Blood flows through the SCD across the dialysate ports and circulates outside the hollow fibers to allow adhesion of activated leukocytes (Fig. 5).

In a single-arm study of 35 patients with CRRT-dependent AKI, the use of SCD resulted in lower-thanexpected mortality rate with dialysis-independence 
among all survivors [77]. The same investigators eventually conducted a randomized controlled trial and compared 69 patients who received CRRT alone to 65 patients who received CRRT + SCD [78]. Participating centers utilized the same CRRT machine (Braun Diapact) but implemented their own citrate anticoagulation protocol and CRRT modality of choice. There was no difference in the 60-day mortality rate $(p=0.23)$, but only about a third of patients achieved the target postfilter ionized calcium goal of $<0.4 \mathrm{mmol} / \mathrm{L}$. In this subgroup of patients, the mortality rate was $16 \%$ in the CRRT + SCD group compared to $41 \%$ in the CRRTalone group. More studies are required to examine any potential survival benefit with this promising device.

\section{Conclusion}

The development of multiple organ support devices in the ICU renders the care of severely ill patients more complex and comprehensive. The nephrologist commonly encounters such patients with one or more mechanical support devices. Understanding the basics of such devices equips the nephrologist with the knowledge required to practice true interdisciplinary care in the intensive care setting.

\section{Disclosure Statement}

The authors have no conflicts of interest to disclose.

\section{References}

1 Ostermann M, Chang RW: Acute kidney injury in the intensive care unit according to RIFLE. Crit Care Med 2007;35:1837-1843; quiz 1852

2 Bagshaw SM, George C, Bellomo R; ANZICS Database Management Committe: A comparison of the RIFLE and AKIN criteria for acute kidney injury in critically ill patients. Nephrol Dial Transplant 2008;23:1569-1574.

3 Thakar CV, Christianson A, Freyberg R, Almenoff P, Render ML: Incidence and outcomes of acute kidney injury in intensive care units: a Veterans Administration study. Crit Care Med 2009;37:2552-2558.

4 Mehta RL, Pascual MT, Soroko S, et al: Spectrum of acute renal failure in the intensive care unit: the PICARD experience. Kidney Int 2004;66:1613-1621.

5 Gibbon JH Jr: Application of a mechanical heart and lung apparatus to cardiac surgery. Minn Med 1954;37:171-185.

6 Hill JD, O’Brien TG, Murray JJ, et al: Prolonged extracorporeal oxygenation for acute post-traumatic respiratory failure (shocklung syndrome). Use of the Bramson membrane lung. N Engl J Med 1972;286:629-634.

7 Bartlett RH, Gazzaniga AB, Fong SW, Burns NE: Prolonged extracorporeal cardiopulmonary support in man. J Thorac Cardiovasc Surg 1974;68:918-932.

8 ECLS Registry Report. July 2016. https:// www.elso.org/Registry/Statistics/InternationalSummary.aspx.

9 Peek GJ, Mugford M, Tiruvoipati R, et al: Efficacy and economic assessment of conventional ventilatory support versus extracorporeal membrane oxygenation for severe adult respiratory failure (CESAR): a multicentre randomised controlled trial. Lancet 2009;374: 1351-1363.

10 Australia, New Zealand Extracorporeal Membrane Oxygenation (ANZ ECMO) Influenza
Investigators, Davies A, et al: Extracorporeal membrane oxygenation for 2009 influenza $\mathrm{A}(\mathrm{H} 1 \mathrm{~N} 1)$ acute respiratory distress syndrome. JAMA 2009;302:1888-1895.

11 ELSO Guidelines for Adult Respiratory Failure v1.3. December 2013. https://www.elso. org/Resources/Guidelines.aspx.

12 Brogan TV, Thiagarajan RR, Rycus PT, Bartlett RH, Bratton SL: Extracorporeal membrane oxygenation in adults with severe respiratory failure: a multi-center database. Intensive Care Med 2009;35:2105-2114.

13 Beiderlinden M, Eikermann M, Boes T, Breitfeld C, Peters J: Treatment of severe acute respiratory distress syndrome: role of extracorporeal gas exchange. Intensive Care Med 2006;32:1627-1631.

14 Schmidt M, Zogheib E, Roze H, et al: The PRESERVE mortality risk score and analysis of long-term outcomes after extracorporeal membrane oxygenation for severe acute respiratory distress syndrome. Intensive Care Med 2013;39:1704-1713.

15 Schmidt M, Bailey M, Sheldrake J, et al: Predicting survival after extracorporeal membrane oxygenation for severe acute respiratory failure. The Respiratory Extracorporeal Membrane Oxygenation Survival Prediction (RESP) score. Am J Respir Crit Care Med 2014;189:1374-1382.

16 Bermudez CA, Rocha RV, Sappington PL, Toyoda Y, Murray HN, Boujoukos AJ: Initial experience with single cannulation for venovenous extracorporeal oxygenation in adults. Ann Thorac Surg 2010;90:991-995.

17 Wang D, Zhou X, Liu X, Sidor B, Lynch J, Zwischenberger JB: Wang-Zwische double lumen cannula-toward a percutaneous and ambulatory paracorporeal artificial lung. ASAIO J 2008;54:606-611.

18 Ius F, Sommer W, Tudorache I, et al: Venoveno-arterial extracorporeal membrane oxy- genation for respiratory failure with severe haemodynamic impairment: technique and early outcomes. Interact Cardiovasc Thorac Surg 2015;20:761-767.

19 Sklar MC, Sy E, Lequier L, Fan E, Kanji HD: Anticoagulation practices during venovenous extracorporeal membrane oxygenation for respiratory failure. A systematic review. Ann Am Thorac Soc 2016;13:22422250.

20 Zapol WM, Snider MT, Hill JD, et al: Extracorporeal membrane oxygenation in severe acute respiratory failure. A randomized prospective study. JAMA 1979;242:21932196.

21 Morris AH, Wallace CJ, Menlove RL, et al: Randomized clinical trial of pressure-controlled inverse ratio ventilation and extracorporeal $\mathrm{CO} 2$ removal for adult respiratory distress syndrome. Am J Respir Crit Care Med 1994;149(2 pt 1):295-305.

$22 \mathrm{https} / / /$ clinicaltrials.gov/ct2/show/ NCT01470703.

23 Moravec R, Neitzel T, Stiller M, et al: First experiences with a combined usage of veno-arterial and veno-venous ECMO in therapy-refractory cardiogenic shock patients with cerebral hypoxemia. Perfusion 2014;29:200-209.

24 Hendrickson SC, Glower DD: A method for perfusion of the leg during cardiopulmonary bypass via femoral cannulation. Ann Thorac Surg 1998;65:1807-1808.

25 Madershahian N, Nagib R, Wippermann J, Strauch J, Wahlers T: A simple technique of distal limb perfusion during prolonged femoro-femoral cannulation. J Card Surg 2006;21: $168-169$.

26 Koeckert MS, Jorde UP, Naka Y, Moses JW, Takayama H: Impella LP 2.5 for left ventricular unloading during venoarterial extracorporeal membrane oxygenation support. J Card Surg 2011;26:666-668. 
27 Weymann A, Schmack B, Sabashnikov A, et al: Central extracorporeal life support with left ventricular decompression for the treatment of refractory cardiogenic shock and lung failure. J Cardiothorac Surg 2014;9:60.

28 Ma P, Zhang Z, Song T, et al: Combining ECMO with IABP for the treatment of critically Ill adult heart failure patients. Heart Lung Circ 2014;23:363-368.

29 Lin CY, Chen YC, Tsai FC, et al: RIFLE classification is predictive of short-term prognosis in critically ill patients with acute renal failure supported by extracorporeal membrane oxygenation. Nephrol Dial Transplant 2006; 21:2867-2873.

30 Yan X, Jia S, Meng X, et al: Acute kidney injury in adult postcardiotomy patients with extracorporeal membrane oxygenation: evaluation of the RIFLE classification and the Acute Kidney Injury Network criteria. Eur J Cardiothorac Surg 2010;37:334-338.

31 Kielstein JT, Heiden AM, Beutel G, et al: Renal function and survival in 200 patients undergoing ECMO therapy. Nephrol Dial Transplant 2013;28:86-90.

32 Han SS, Kim HJ, Lee SJ, et al: Effects of renal replacement therapy in patients receiving extracorporeal membrane oxygenation: a metaanalysis. Ann Thorac Surg 2015;100:14851495.

33 Askenazi DJ, Selewski DT, Paden ML, et al: Renal replacement therapy in critically ill patients receiving extracorporeal membrane oxygenation. Clin J Am Soc Nephrol 2012;7: 1328-1336.

34 Villa G, Katz N, Ronco C: Extracorporeal membrane oxygenation and the kidney. Cardiorenal Med 2015;6:50-60.

35 Bouchard J, Soroko SB, Chertow GM, et al: Fluid accumulation, survival and recovery of kidney function in critically ill patients with acute kidney injury. Kidney Int 2009;76:422-427.

36 Fleming GM, Askenazi DJ, Bridges BC, et al A multicenter international survey of renal supportive therapy during ECMO: the kidney intervention during extracorporeal membrane oxygenation (KIDMO) group. ASAIO J 2012;58:407-414.

37 Schmidt M, Bailey M, Kelly J, et al: Impact of fluid balance on outcome of adult patients treated with extracorporeal membrane oxygenation. Intensive Care Med 2014;40:12561266.

38 National Heart, Lung, and Blood Institute Acute Respiratory Distress Syndrome (ARDS) Clinical Trials Network, Wiedemann HP, et al: Comparison of two fluid-management strategies in acute lung injury. $\mathrm{N}$ Engl J Med 2006;354:2564-2575.

39 Cerda J, Tolwani AJ, Warnock DG: Critical care nephrology: management of acid-base disorders with CRRT. Kidney Int 2012;82:918.

40 Kallet RH, Liu K, Tang J: Management of acidosis during lung-protective ventilation in acute respiratory distress syndrome. Respir Care Clin N Am 2003;9:437-456.
41 Acute Respiratory Distress Syndrome Network, Brower RG, Matthay MA, et al: Ventilation with lower tidal volumes as compared with traditional tidal volumes for acute lung injury and the acute respiratory distress syndrome. N Engl J Med 2000;342:13011308.

42 Rubenfeld GD, Cooper C, Carter G, Thompson BT, Hudson LD: Barriers to providing lung-protective ventilation to patients with acute lung injury. Crit Care Med 2004;32: 1289-1293.

43 Paden ML, Warshaw BL, Heard ML, Fortenberry JD: Recovery of renal function and survival after continuous renal replacement therapy during extracorporeal membrane oxygenation. Pediatr Crit Care Med 2011;12: 153-158.

44 Meyer RJ, Brophy PD, Bunchman TE, et al: Survival and renal function in pediatric patients following extracorporeal life support with hemofiltration. Pediatr Crit Care Med 2001;2:238-242.

45 Hoover NG, Heard M, Reid C, et al: Enhanced fluid management with continuous venovenous hemofiltration in pediatric respiratory failure patients receiving extracorporeal membrane oxygenation support. Intensive Care Med 2008;34:2241-2247.

46 Santiago MJ, Sanchez A, Lopez-Herce J, et al: The use of continuous renal replacement therapy in series with extracorporeal membrane oxygenation. Kidney Int 2009;76:1289-1292.

47 Sucosky P, Dasi LP, Paden ML, Fortenberry JD, Yoganathan AP: Assessment of current continuous hemofiltration systems and development of a novel accurate fluid management system for use in extracorporeal membrane oxygenation. J Med Devices 2008;2:035002.

48 Kormos RL, Teuteberg JJ, Pagani FD, et al: Right ventricular failure in patients with the HeartMate II continuous-flow left ventricular assist device: incidence, risk factors, and effect on outcomes. J Thorac Cardiovasc Surg 2010; 139:1316-1324

49 Kazui T, Tran PL, Echeverria A, et al: Minimally invasive approach for percutaneous CentriMag right ventricular assist device support using a single PROTEKDuo Cannula. J Cardiothorac Surg 2016;11:123.

50 Takeda K, Naka Y, Yang JA, et al: Outcome of unplanned right ventricular assist device support for severe right heart failure after implantable left ventricular assist device insertion. J Heart Lung Transplant 2014;33:141-148.

51 Shen TC, Tsai KT, Hu CY, Chen RJ: Skirted cannula technique for apical cannulation in implantation of centrimag left ventricular assist device. Ann Thorac Surg 2016;101:24042406.

52 Takayama H, Soni L, Kalesan B, et al: Bridgeto-decision therapy with a continuous-flow external ventricular assist device in refractory cardiogenic shock of various causes. Circ Heart Fail 2014;7:799-806

53 Thiele H, Sick P, Boudriot E, et al: Randomized comparison of intra-aortic balloon sup- port with a percutaneous left ventricular assist device in patients with revascularized acute myocardial infarction complicated by cardiogenic shock. Eur Heart J 2005;26: 1276-1283.

54 Kapur NK, Paruchuri V, Korabathina R, et al: Effects of a percutaneous mechanical circulatory support device for medically refractory right ventricular failure. J Heart Lung Transplant 2011;30:1360-1367.

55 Kiernan MS, Krishnamurthy B, Kapur NK: Percutaneous right ventricular assist via the internal jugular vein in cardiogenic shock complicating an acute inferior myocardial infarction. J Invasive Cardiol 2010;22:E23E26.

56 Lee WM: Acute liver failure. N Engl J Med 1993;329:1862-1872.

57 Lee WM, Squires RH Jr, Nyberg SL, Doo E, Hoofnagle JH: Acute liver failure: summary of a workshop. Hepatology 2008;47:1401-1415.

58 Munoz SJ: Difficult management problems in fulminant hepatic failure. Semin Liver Dis 1993;13:395-413.

59 Caraceni P, Van Thiel DH: Acute liver failure. Lancet 1995;345:163-169.

60 Barshes NR, Gay AN, Williams B, Patel AJ, Awad SS: Support for the acutely failing liver: a comprehensive review of historic and contemporary strategies. J Am Coll Surg 2005; 201:458-476.

61 Park JK, Lee DH: Bioartificial liver systems: current status and future perspective. J Biosci Bioeng 2005;99:311-319.

62 Nevens F, Laleman W: Artificial liver support devices as treatment option for liver failure. Best Pract Res Clin Gastroenterol 2012;26: $17-26$.

63 Stange J, Ramlow W, Mitzner S, Schmidt R Klinkmann H: Dialysis against a recycled albumin solution enables the removal of albumin-bound toxins. Artif Organs 1993;17: 809-813.

64 Banares R, Nevens F, Larsen FS, et al: Extracorporeal albumin dialysis with the molecular adsorbent recirculating system in acute-onchronic liver failure: the RELIEF trial. Hepatology 2013;57:1153-1162

65 Falkenhagen D, Strobl W, Vogt G, et al: Fractionated plasma separation and adsorption system: a novel system for blood purification to remove albumin bound substances. Artif Organs 1999;23:81-86.

66 Kribben A, Gerken G, Haag S, et al: Effects of fractionated plasma separation and adsorption on survival in patients with acute-onchronic liver failure. Gastroenterology 2012; 142:782-789.e783.

67 Krisper P, Haditsch B, Stauber R, et al: In vivo quantification of liver dialysis: comparison of albumin dialysis and fractionated plasma separation. J Hepatol 2005;43:451-457.

68 Sauer IM, Goetz M, Steffen I, et al: In vitro comparison of the molecular adsorbent recirculation system (MARS) and single-pass albumin dialysis (SPAD). Hepatology 2004;39: 1408-1414. 
69 Sponholz C, Matthes K, Rupp D, et al: Molecular adsorbent recirculating system and single-pass albumin dialysis in liver failure - a prospective, randomised crossover study. Crit Care 2016;20:2.

70 Benyoub K, Muller $\mathrm{M}$, Bonnet $\mathrm{A}$, et al: Amounts of bile acids and bilirubin removed during single-pass albumin dialysis in patients with liver failure. Ther Apher Dial 2011; 15:504-506.

71 Chawla LS, Georgescu F, Abell B, Seneff MG, Kimmel PL: Modification of continuous venovenous hemodiafiltration with single-pass albumin dialysate allows for removal of serum bilirubin. Am J Kidney Dis 2005;45:e51-e56.

72 Askenazi DJ, Goldstein SL, Chang IF, Elenberg E, Feig DI: Management of a severe car- bamazepine overdose using albumin-enhanced continuous venovenous hemodialysis. Pediatrics 2004;113:406-409.

73 Chan WK, Hui WF: Sequential use of hemoperfusion and single-pass albumin dialysis can safely reverse methotrexate nephrotoxicity. Pediatr Nephrol 2016;31:1699-1703.

74 Churchwell MD, Pasko DA, Smoyer WE Mueller BA: Enhanced clearance of highly protein-bound drugs by albumin-supplemented dialysate during modeled continuous hemodialysis. Nephrol Dial Transplant 2009; 24:231-238.

75 Ding F, Song JH, Jung JY, et al: A biomimetic membrane device that modulates the excessive inflammatory response to sepsis. PLoS One 2011;6:e18584.
76 Szamosfalvi B, Westover A, Buffington D, Yevzlin A, Humes HD: Immunomodulatory device promotes a shift of circulating monocytes to a less inflammatory phenotype in chronic hemodialysis patients. ASAIO J 2016; 62:623-630.

77 Tumlin JA, Chawla L, Tolwani AJ, et al: The effect of the selective cytopheretic device on acute kidney injury outcomes in the intensive care unit: a multicenter pilot study. Semin Dial 2013;26:616-623.

78 Tumlin JA, Galphin CM, Tolwani AJ, et al: A multi-center, randomized, controlled, pivotal study to assess the safety and efficacy of a selective cytopheretic device in patients with acute kidney injury. PLoS One 2015;10: e0132482. 